\title{
ORDER REDUCTION, PROJECTABILITY AND CONSTRAINTS OF SECOND-ORDER FIELD THEORIES AND HIGHER-ORDER MECHANICS
}

\author{
Jordi Gaset, NARCiso Román-Roy \\ Department of Mathematics. Ed. C-3, Campus Norte UPC \\ C/ Jordi Girona 1. 08034 Barcelona. Spain. \\ e-mails: gaset.jordi@gmail.com,narciso.roman@upc.edu
}

October 8, 2018

\begin{abstract}
The projectability of Poincaré-Cartan forms in a third-order jet bundle $J^{3} \pi$ onto a lower-order jet bundle is a consequence of the degenerate character of the corresponding Lagrangian. This fact is analyzed using the constraint algorithm for the associated Euler-Lagrange equations in $J^{3} \pi$. The results are applied to study the Hilbert Lagrangian for the Einstein equations (in vacuum) from a multisymplectic point of view. Thus we show how these equations are a consequence of the application of the constraint algorithm to the geometric field equations, meanwhile the other constraints are related with the fact that this second-order theory is equivalent to a first-order theory. Furthermore, the case of higher-order mechanics is also studied as a particular situation.
\end{abstract}

Key words: 2nd-order Lagrangian field theories, Higher-order mechanics, PoincaréCartan form, Einstein-Hilbert action.

AMS s.c. (2010): 70H50, 53D42, 55R10, 83C05.

\section{Introduction}

There are some models in classical field theories where, as a consequence of the singularity of the Lagrangian, the order of the Euler-Lagrange equations is lower than expected. A geometrical way of understanding this problem is considering the projectability of the higher-order Poincaré-Cartan form onto lower-order jet bundles [3, 13, 14, 16, 17]. We review the conditions for this projectability and 
study their consequences using the constraint algorithm for the field equations of second order (singular) field theories, thus enlarging the results stated in previous papers [3, 9, 14, 16, 17]. This constitutes the main result of the paper and it is stated in Theorem 1 .

In this paper we restrict our study to second order field theories in order to avoid some kinds of problems involving the ambiguity in the definition of the PoincaréCartan form in a higher-order jet bundle, the non-uniqueness of the construction of the Legendre map associated with a higher-order Lagrangian and the choice of the multimomentum phase space for the Hamiltonian formalism [1, 8, 10, 12, 13]. As it is well-known, for the second-order case, all the Poincaré-Cartan forms are proved to be equivalent and the Legendre map and the Hamiltonian multimomentum phase space can be unambiguously defined [15, 18, 19].

As a relevant example, the case of the Hilbert Lagrangian for the Einstein equations with no matter fonts is analyzed. In particular, we show how these equations are obtained as constraints appearing as a consequence of the application of the constraint algorithm to the geometric field equations which are stated in the corresponding third-order jet bundle. The other constraints arising in the algorithm are of geometrical nature. They are related with the fact that we are working with some unnecessary degrees of freedom, because we are using a third-order jet bundle to describe a second-order theory that, as a consequence of the projectibility of the Poincare-Cartan form, is really equivalent to a first-order theory [17. In addition, this study constitutes a new approach to a multisymplectic formulation of the Lagrangian formalism for this model, which is different to other previous attemps on this subject [20].

Finally, this analysis is done for the case of higher-order mechanics which, as it is well-known, can be considered as a particular case of higher-order field theories. Here we consider dynamical systems of any order, since the above-mentioned ambiguities about the construction of the Poincaré-Cartan form and the Legendre map do not occur in higher-order tangent bundles.

All the manifolds are real, second countable and $\mathrm{C}^{\infty}$. The maps and the structures are $\mathrm{C}^{\infty}$. Sum over repeated indices is understood. In order to use coordinate expressions, remember that a multi-index $I$ is an element of $\mathbb{Z}^{m}$ where every component is positive, the $i$ th position of the multi-index is denoted $I(i)$, and $|I|=\sum_{i=1}^{m} I(i)$ is the length of the multi-index. An expression as $|I|=k$ means that the expression is taken for every multi-index of length $k$. Furthermore, the element $1_{i} \in \mathbb{Z}^{m}$ is defined as $1_{i}(j)=\delta_{i}^{j}$. Finally, $n(i j)$ is a combinatorial factor which $n(i j)=1$ for $i=j$, and $n(i j)=2$ for $i \neq j$. 


\section{Order reduction and projectability of the Poincaré- Cartan form}

Let $M$ be an $m$-dimensional manifold and $\pi: E \rightarrow M$ a fiber bundle over $M$ with $\operatorname{dim} E=m+n$ (the configuration bundle of a classical field theory). The $k$-jet manifold of $\pi$ is denoted $J^{k} \pi$ and is endowed with the natural projections $\pi_{s}^{k}: J^{k} \pi \rightarrow$ $J^{s} \pi, \pi^{k}: J^{k} \pi \rightarrow E, \bar{\pi}^{k}: J^{k} \pi \rightarrow M$; for $k>s \geq 0$. Then, a section $\psi: M \rightarrow J^{k} \pi$ of $\bar{\pi}^{k}$ is holonomic if $j^{k}\left(\pi^{k} \circ \psi\right)=\psi$; that is, $\psi$ is the $k$ th prolongation of a section $\phi=\pi^{k} \circ \psi: M \rightarrow E$.

Remember that a form $\omega \in \Omega^{s}(E)$ is said to be $\pi$-semibasic if $i(X) \omega=0$, and $\pi$-basic or $\pi$-projectable if $i(X) \omega=0$ and $\mathrm{L}(X) \omega=0$, for every $\pi$-vertical vector field $X \in \mathfrak{X}^{V}(\pi)$ (here, the symbols $i$ and L denote the inner contraction and the Lie derivative, respectively). As a consequence of Cartan's formula, $\mathrm{L}(X) \omega=$ $i(X) \mathrm{d} \omega+\mathrm{d} i(X) \omega$, a form $\omega \in \Omega^{n}(E)$ is $\pi$-basic if, and only if, $\omega$ and $\mathrm{d} \omega$ are $\pi$ semibasic.

A special kind of vector fields are the coordinate total derivatives [15, 18]:

$$
D_{i}=\frac{\partial}{\partial x^{i}}+\sum_{|I|=0}^{k} u_{I+1_{i}}^{\alpha} \frac{\partial}{\partial u_{I}^{\alpha}} .
$$

For every function $f \in \mathrm{C}^{\infty}\left(J^{k} \pi\right)$, we have that $D_{i} f:=\mathrm{L}\left(D_{i}\right) f \in \mathrm{C}^{\infty}\left(J^{k+1} \pi\right)$. In addition, we have:

- If $X \in \mathfrak{X}^{V}\left(\pi_{s}^{k}\right)$, then $\left[D_{i}, X\right] \in \mathfrak{X}^{V}\left(\pi_{s-1}^{k}\right)$.

- For $f \in C^{\infty}\left(J^{k} \pi\right)$, if $f$ is $\pi_{s}^{k}$-basic then $D_{i} f$ is $\pi_{s+1}^{k}$-basic.

We show some consequences of the projectability of the Poincaré-Cartan form for second order Lagrangian classical field theories. The Lagrangian form that describes the theory is a $\bar{\pi}^{2}$-semibasic m-form $\mathcal{L}=L\left(\bar{\pi}^{2}\right)^{*} \omega \in \Omega^{m}\left(J^{2} \pi\right)$, where $L \in \mathrm{C}^{\infty}\left(J^{2} \pi\right)$ is the Lagrangian function, $\omega$ is the volume form in $M$, and $\bar{\pi}^{2}: J^{2} \pi \rightarrow M$. Natural coordinates of $J^{3} \pi$ adapted to the fibration are $\left(x^{i}, u^{\alpha}, u_{i}^{\alpha}, u_{I}^{\alpha}, u_{J}^{\alpha}\right)$, such that $\omega=$ $\mathrm{d} x^{1} \wedge \ldots \wedge \mathrm{d} x^{m} \equiv \mathrm{d}^{m} x ; 1 \leq i \leq m, 1 \leq \alpha \leq n$, and $I, J$ are multiindices with $|I|=2,|J|=3,[18]$.

The Poincaré-Cartan $m$-form $\Theta_{\mathcal{L}} \in \Omega^{m}\left(J^{3} \pi\right)$ is locally given by

$$
\Theta_{\mathcal{L}}=L_{\alpha}^{i} \mathrm{~d} u^{\alpha} \wedge \mathrm{d}^{m-1} x_{i}+L_{\alpha}^{i j} \mathrm{~d} u_{i}^{\alpha} \wedge \mathrm{d}^{m-1} x_{j}+\left(L-L_{\alpha}^{i} u_{i}^{\alpha}-L_{\alpha}^{i j} u_{1_{i}+1_{j}}^{\alpha}\right) \mathrm{d}^{m} x,
$$

where $\mathrm{d}^{m-1} x_{j}=i\left(\frac{\partial}{\partial x^{j}}\right) \mathrm{d}^{m} x$ and the functions $L_{\alpha}^{i}, L_{\alpha}^{i j} \in C^{\infty}\left(J^{3} \pi\right)$ are

$$
L_{\alpha}^{i}=\frac{\partial L}{\partial u_{i}^{\alpha}}-D_{j} L_{\alpha}^{i j} \quad ; \quad L_{\alpha}^{i j}=\frac{1}{n(i j)} \frac{\partial L}{\partial u_{1_{i}+1_{j}}^{\alpha}} .
$$


Lemma 1. For $s=1,2$, the following conditions are equivalent:

1. $\Theta_{\mathcal{L}}$ projects onto $J^{s} \pi$.

2. $\mathrm{d} \Theta_{\mathcal{L}}$ is $\pi_{s}^{3}$-semibasic.

3. $\mathrm{L}(X) L_{\alpha}^{i}=0$ and $\mathrm{L}(X) L_{\alpha}^{i j}=0$; for every $X \in \mathfrak{X}^{V}\left(\pi_{s}^{3}\right)$.

(Proof): $\quad(1 \Leftrightarrow 2)$ is a consequence of Cartan's formula.

For $(2 \Leftrightarrow 3)$, in the case $s=2$, we compute the condition 2 in coordinates. It turns to be equivalent to

$$
\frac{\partial L_{\alpha}^{i}}{\partial u_{J}^{\beta}}=0 \quad, \quad \frac{\partial L_{\alpha}^{i j}}{\partial u_{J}^{\beta}}=0 \quad, \quad \frac{\partial}{\partial u_{J}^{\beta}}\left(L-L_{\alpha}^{i} u_{i}^{\alpha}-L_{\alpha}^{i j} u_{1_{i}+1_{j}}^{\alpha}\right)=0 \quad ;
$$

(for $|J|=3$, and for every $\beta, \alpha, i$ and $j$ ). The last equation is a consequence of the other two (because $L$ does not depend on $u_{J}^{\beta}$ ); which are locally equivalent to 3 , since $\left\{\frac{\partial}{\partial u_{J}^{\beta}}\right\}$ generates $\mathfrak{X}^{V}\left(\pi_{s}^{3}\right)$. The case $s=1$ can be proved in a similar way.

Other important results concerning to this topic (that we present here for completeness) are the following [16]:

Proposition 1. If $\Theta_{\mathcal{L}}$ projects onto $J^{s} \pi$, then the order of the Euler-Lagrange equations is at most $s+1$.

Proposition 2. If there exist $\mathcal{L}^{\prime} \in \Omega^{m}\left(J^{1} \pi\right)$ such that $\Theta_{\mathcal{L}}=\left(\pi_{1}^{3}\right)^{*} \Theta_{\mathcal{L}^{\prime}}$, then $\mathcal{L}=$ $\left(\pi_{1}^{3}\right)^{*} \mathcal{L}^{\prime}$

Concerning to the last proposition, the study of the existence of an equivalent lower order Lagrangian $\mathcal{L}^{\prime} \in \Omega^{m}\left(J^{1} \pi\right)$ has been analysed in [3, 17].

If the Poincaré-Cartan form $\Theta_{\mathcal{L}}$ projects onto a lower-order jet bundle, it is associated to a highly degenerate Lagrangian (this is just a consequence of the third item in Lemma 1). As a consequence of this fact, the field equations could not have admissible solutions everywhere in $J^{3} \pi$, but in some submanifold of it which can be obtained after applying a suitable constraint algorithm (see, for instance, 4]).

In order to study these facts, we introduce the following concepts [7]:

Definition 1. An $m$-multivector field in $J^{3} \pi$ is a skew-symmetric contravariant tensor of order $m$ in $J^{3} \pi$. The set of $m$-multivector fields in $J^{3} \pi$ is denoted $\mathfrak{X}^{m}\left(J^{3} \pi\right)$.

A multivector field $\mathbf{X} \in \mathfrak{X}^{m}\left(J^{3} \pi\right)$ is said to be locally decomposable if, for every $p \in J^{3} \pi$, there is an open neighbourhood $U_{p} \subset J^{3} \pi$ and $X_{1}, \ldots, X_{m} \in \mathfrak{X}\left(U_{p}\right)$ such that $\left.\mathbf{X}\right|_{U_{p}}=X_{1} \wedge \ldots \wedge X_{m}$. 
Non-vanishing locally decomposable $m$-multivector fields $\mathbf{X} \in \mathfrak{X}^{m}\left(J^{3} \pi\right)$ are locally associated with $m$-dimensional distributions $D \subset \mathrm{T} J^{3} \pi$. Then, $\mathbf{X}$ is integrable if its associated distribution is integrable. In particular, $\mathbf{X}$ is holonomic if it is integrable and its integral sections are holonomic sections of $\bar{\pi}^{3}$.

Then, the solutions to the Euler-Lagrange equations for a second-order field theory are the integral sections of locally decomposable holonomic multivector fields $\mathbf{X} \in \mathfrak{X}^{m}\left(J^{3} \pi\right)$ such that

$$
i(\mathbf{X}) \mathrm{d} \Theta_{\mathcal{L}}=0
$$

Therefore:

Theorem 1. If $\Theta_{\mathcal{L}}$ projects onto $J^{s} \pi$, then solutions to the corresponding EulerLagrange equations only exist in the points of a submanifold $\mathcal{S} \hookrightarrow J^{3} \pi$, where $\mathcal{S}$ is locally defined by the constraint functions given by

- $L_{\alpha}^{0}=0$; if $s=2$.

- $L_{\alpha}^{0}=0$ and $D_{i} L_{\alpha}^{0}=0$; if $s=1$.

Where $L_{\alpha}^{0}=\frac{\partial L}{\partial u^{\alpha}}-D_{i} L_{\alpha}^{i}=\frac{\partial L}{\partial u^{\alpha}}-D_{i} \frac{\partial L}{\partial u_{i}^{\alpha}}+D_{I} \frac{\partial L}{\partial u_{I}^{\alpha}}$.

(Proof): $\mathbf{X}$ can be written in coordinates as

$$
\mathbf{X}=f \bigwedge_{i=1}^{m}\left(D_{i}+\left(F_{J, i}^{\alpha}-u_{J+1_{i}}^{\alpha}\right) \frac{\partial}{\partial u_{J}^{\alpha}}\right)=f \bigwedge_{i=1}^{m} X_{i} ;
$$

for $f, F_{J, i}^{\alpha} \in C^{\infty}\left(J^{3} \pi\right),(|J|=3)$. Using this expression, equation (2) reduces to

$$
L_{\alpha}^{0}+\left(F_{J, i}^{\beta}-u_{J+1_{i}}^{\beta}\right) \frac{\partial L_{\alpha}^{i}}{\partial u_{J}^{\beta}}=0
$$

which are the Euler-Lagrange equations for multivector fields. If $\Theta_{\mathcal{L}}$ projects either onto $J^{1} \pi$ or $J^{2} \pi$, by Lemma 1 we have $\frac{\partial L_{\alpha}^{i}}{\partial u_{J}^{\beta}}=0$, and then from (13) we get $L_{\alpha}^{0}=$ 0 . Observe that, as a consequence, we cannot compute any of the functions $F_{J, i}^{\alpha}$. Actually $L_{\alpha}^{0}=0$ are restrictions for the points of the manifold $J^{3} \pi$, which we assume that define a submanifold $\mathcal{S}_{1} \subset J^{3} \pi$, where the equation (2) have solutions. In order to find $F_{J, i}^{\alpha}$ we use the constraint algorithm (as it is outlined, for instance, in [15]). So we look for the points of $\mathcal{S}_{1}$ where the multivector fields which are solutions to (2) (on $\mathcal{S}_{1}$ ) are tangent to $\mathcal{S}_{1}$. Thus, imposing this consistency or tangency condition we get

$$
0=\mathrm{L}\left(X_{i}\right) L_{\alpha}^{0}=D_{i} L_{\alpha}^{0}+\left(F_{J, i}^{\beta}-u_{J+1_{i}}^{\beta}\right) \frac{\partial L_{\alpha}^{0}}{\partial u_{J}^{\beta}} \quad\left(\text { on } \mathcal{S}_{1}\right)
$$


If $\Theta_{\mathcal{L}}$ projects onto $J^{1} \pi$, then the associated Euler-Lagrange equations are of order at most 2 (by proposition 11). This implies that $L_{\alpha}^{0}$, which are the Euler-Lagrange equations before being evaluatedon sections, are $\pi_{2}^{3}$-projectable. Thus, $\frac{\partial L_{\alpha}^{0}}{\partial u_{J}^{\beta}}=0$, and we find new restrictions, $D_{i} L_{\alpha}^{0}=0$ which are assumed to define a new submanifold $\mathcal{S}_{2} \subset \mathcal{S}_{1} \subset J^{3} \pi$ where the solutions to (2) are tangent to $\mathcal{S}_{1}$.

Notice that, depending on the Lagrangian, we may need to continue the constraint algorithm, so obtaining that

$$
D_{j} D_{i} L_{\alpha}^{0}+\left(F_{J, j}^{\beta}-u_{J+1_{j}}^{\beta}\right) \frac{\partial D_{i} L_{\alpha}^{0}}{\partial u_{J}^{\beta}}=0 \quad\left(\text { on } \mathcal{S}_{2}\right)
$$

This process continues until the new conditions hold identically and we find a final constraint submanifold $\mathcal{S}_{f}$ of $J^{3} \pi$ where solutions to (2) are tangent to $\mathcal{S}_{f}$.

\section{The Hilbert-Einstein Lagrangian}

Here $M$ is a 4-manifold representing space-time and the fibers are the spaces of Lorentzian metrics. The fiber coordinates in $E$ are $\left(x^{\mu}, g_{\mu \nu}\right)(\mu, \nu$ and all greek indices in this section run from 0 to 3 ), where $g_{\mu \nu}$ are the component functions of the metric. The Hilbert Lagrangian function without matter is:

$$
L=\sqrt{|\operatorname{det}(g)|} R=\sqrt{|\operatorname{det}(g)|} g^{\mu \nu} R_{\mu \nu},
$$

where $R=g^{\mu \nu} R_{\mu \nu}$ is the scalar curvature, $R_{\mu \nu}=D_{\rho} \Gamma_{\mu \nu}^{\rho}-D_{\mu} \Gamma_{\rho \nu}^{\rho}+\Gamma_{\mu \nu}^{\rho} \Gamma_{\delta \rho}^{\delta}-\Gamma_{\delta \nu}^{\rho} \Gamma_{\mu \rho}^{\delta}$ are the components of the Ricci tensor, $\Gamma_{\mu \nu}^{\rho}=\frac{1}{2} g^{\rho \lambda}\left(\frac{\partial g_{\nu \lambda}}{\partial x^{\mu}}+\frac{\partial g_{\lambda \mu}}{\partial x^{\nu}}-\frac{\partial g_{\mu \nu}}{\partial x^{\lambda}}\right)$ are the Christoffel symbols of the Levi-Civita connection of $g$, and $g^{\mu \nu}$ denotes the inverse matrix of $g$, namely: $g^{\mu \nu} g_{\nu \rho}=\delta_{\rho}^{\mu}$. As the Christoffel symbols depend on firstorder derivatives of $g_{\mu \nu}$ and taking into account the expression (1) we have that $R$ contains second-order derivatives of the components of the metric and thus this is a second-order field theory.

The Poincaré-Cartan form $\Theta_{\mathcal{L}}$ associated with the Hilbert Lagrangian density $\mathcal{L}=L\left(\bar{\pi}^{2}\right)^{*} \omega=L \mathrm{~d}^{4} x$ is

$$
\begin{aligned}
\Theta_{\mathcal{L}} & =-\left(\sum_{\alpha \leq \beta} L^{\alpha \beta, \mu} g_{\alpha \beta, \mu}+\sum_{\alpha \leq \beta} L^{\alpha \beta, I} g_{\alpha \beta, I}-\sum_{\alpha \leq \beta} L\right) \mathrm{d}^{4} x \\
& +\sum_{\alpha \leq \beta} L^{\alpha \beta, \mu} \mathrm{d} g_{\alpha \beta} \wedge \mathrm{d}^{m-1} x_{\mu}+\sum_{\alpha \leq \beta} L^{\alpha \beta, \mu \nu} \mathrm{d} g_{\alpha \beta, \mu} \wedge \mathrm{d}^{m-1} x_{\nu}
\end{aligned}
$$


where

$$
\begin{aligned}
L^{\alpha \beta, \mu} & =\frac{\partial L}{\partial g_{\alpha \beta, \mu}}-\sum_{\nu=0}^{3} \frac{1}{n(\mu \nu)} D_{\nu}\left(\frac{\partial L}{\partial g_{\alpha \beta, \mu \nu}}\right) \\
& =\frac{n(\alpha \beta)}{2} \sqrt{|\operatorname{det}(g)|}\left(\Gamma_{\nu \sigma}^{\alpha}\left(g^{\beta \sigma} g^{\mu \nu}-g^{\beta \mu} g^{\sigma \nu}\right)+\Gamma_{\nu \sigma}^{\beta}\left(g^{\alpha \sigma} g^{\mu \nu}-g^{\alpha \mu} g^{\sigma \nu}\right)\right) \\
L^{\alpha \beta, \mu \nu} & =\frac{1}{n(\mu \nu)} \frac{\partial L}{\partial g_{\alpha \beta, \mu \nu}}=\frac{n(\alpha \beta)}{2} \sqrt{|\operatorname{det}(g)|}\left(g^{\alpha \mu} g^{\beta \nu}+g^{\alpha \nu} g^{\beta \mu}-2 g^{\alpha \beta} g^{\mu \nu}\right) .
\end{aligned}
$$

This form projects onto $J^{1} \pi$ and hence the propositions of section 2 hold. As is well known, the corresponding Euler-Lagrange equations, which are essentially the Einstein equations [2], are of second order.

Moreover, as it is noted in [16], the projected form $\left(\pi_{1}^{3}\right)^{*} \Theta_{\mathcal{L}}$ is not the PoincaréCartan form of any Lagrangian of order 1 . Nevertheless, there exists a Lagrangian of order 1 whose Euler-Lagrange equations have solutions which are the same than those for the Hilbert Lagrangian [3, 17].

Finally, we apply in detail the theorem 1 to the Hilbert Lagrangian (that is, the constraint algorithm). The local expression of a holonomic and locally decomposable multivector field in $J^{3} \pi$ is

$$
\mathbf{X}_{\mathcal{L}}=\bigwedge_{\rho=0}^{3} X_{\rho}=\bigwedge_{\rho=0}^{3}\left(D_{\rho}+\sum_{\alpha \leq \beta}\left(F_{\alpha \beta ; J, \rho}-g_{\alpha \beta ; J+1_{\rho}}\right) \frac{\partial}{\partial g_{\alpha \beta ; J}}\right)
$$

and the equations (2) take the local expression:

$$
\begin{aligned}
D_{\mu} L^{\alpha \beta, \mu}-\frac{\partial L}{\partial g_{\alpha \beta}} & =0 \\
D_{\nu} L^{\alpha \beta, \mu \nu}+L^{\alpha \beta, \mu}-\frac{\partial L}{\partial g_{\alpha \beta, \mu}} & =0 \\
n(\mu \nu) n(\alpha \beta) L^{\alpha \beta, \mu \nu}-n(\alpha \beta) \frac{\partial L}{\partial g_{\alpha \beta, \mu \nu}} & =0 .
\end{aligned}
$$

The equations (7) and (8) are just the identities (44) and (5) . Furthermore, using (4) we see that equations (6) are:

$$
0=\frac{\partial L}{\partial g_{\alpha \beta}}-D_{\mu} \frac{\partial L}{\partial g_{\alpha \beta, \mu}}+D_{I} \frac{\partial L}{\partial g_{\alpha \beta, I}}=-\sqrt{|\operatorname{det}(g)|} n(\alpha \beta)\left(R^{\alpha \beta}-\frac{1}{2} g^{\alpha \beta} R\right) \equiv L^{\alpha \beta} .
$$

Notice that with these equations we cannot determine any of the unknows $F_{\alpha \beta ; J, \rho}$. Actually $L^{\alpha \beta}$ project onto $J^{2} \pi$; hence they do not depend on the higher-order derivatives and therefore $L^{\alpha \beta}=0$ are constraints which define the submanifold $\mathcal{S}_{1} \subset J^{3} \pi$. These functions, evaluated on the points of holonomic sections of $\bar{\pi}^{3}$ are the EulerLagrange equations; that is, they give the Einstein equations

$$
R^{\alpha \beta}-\frac{1}{2} g^{\alpha \beta} R=0
$$


which, in this way, turn out to be constraints defining the submanifold $\mathcal{S}_{1}$. The tangency conditions for these functions $L^{\alpha \beta}$ lead to

$$
\mathrm{L}\left(X_{\rho}\right) L^{\alpha \beta}=D_{\rho} L^{\alpha \beta}+\sum_{\mu \leq \nu}\left(F_{\mu \nu ; J, \rho}-g_{\mu \nu ; J+1_{\rho}}\right) \frac{\partial L^{\alpha \beta}}{\partial g_{\mu \nu ; J}}=D_{\rho} L^{\alpha \beta}=0 \quad\left(\text { on } \mathcal{S}_{1}\right),
$$

since $\frac{\partial L^{\alpha \beta}}{\partial g_{\mu \nu ; J}}=0$. By the properties of the total derivative, we have that the functions $D_{\rho} L^{\alpha \beta}$ project onto $J^{3} \pi$ and then the functions $D_{\rho} L^{\alpha \beta}$ are constraints again and define the submanifold $\mathcal{S}_{2} \subset \mathcal{S}_{1} \subset J^{3} \pi$ (this is also obvious bearing in mind (11)). Finally, the new tangency conditions lead to the equalities

$$
D_{\tau} D_{\rho} L^{\alpha \beta}+\sum_{\mu \leq \nu}\left(F_{\mu \nu ; J, \tau}-g_{\mu \nu ; J+1_{\tau}}\right) \frac{\partial D_{\rho} L^{\alpha \beta}}{\partial g_{\mu \nu ; J}}=0 \quad\left(\text { on } \mathcal{S}_{2}\right)
$$

which are not constraints since they contain the unknown functions $F_{\mu \nu ; J, \tau}$.

In order to understand the implications of equations (10) and (11), consider an holonomic section $\psi: M \rightarrow J^{3} \pi$. When evaluated at the section, they look:

$$
\left.\left(D_{\rho} L^{\alpha \beta}\right)\right|_{\psi}=\frac{\partial\left(L^{\alpha \beta} \circ \psi\right)}{\partial x^{\rho}}=0 \quad,\left.\quad\left(D_{\tau} D_{\rho} L^{\alpha \beta}\right)\right|_{\psi}=\frac{\partial^{2}\left(L^{\alpha \beta} \circ \psi\right)}{\partial x^{\tau} \partial x^{\rho}}=0 .
$$

Here we have used that, if $\psi$ is an integral section of $\mathbf{X}$, then $\left.\left(F_{\mu \nu ; J, \tau}-g_{\mu \nu ; J+1_{\tau}}\right)\right|_{\psi}=$ 0 . So, if $\psi$ is a solution to the Einstein equations (that is, $L^{\alpha \beta} \circ \psi=0$ ), then $\psi$ also satisfies equations (10) and (11). Therefore, from the physical point of view, the only relevant equations are (9), which are equivalent to the Einstein equations. The other equations (10) and (11) contain no physical information: they are of geometrical nature. They arise from the fact that we are using a third-order jet bundle $J^{3} \pi$, prepared for describing a second-order theory, for a Lagrangian which is physically equivalent to a first-order Lagrangian and hence, we have redundant information.

In a further paper, Hilbert's Lagrangian as well as other Lagrangian models for gravitation will be studied in detail using this procedure and the unified formalism developed in [15].

\section{Application to higher-order mechanics}

Now, consider the particular case where $\pi: E \rightarrow \mathbb{R}$, with $\operatorname{dim} E=n+1$, is the configuration bundle of a higher-order non-autonomous theory. We have the natural projections $\pi_{s}^{k}: J^{k} \pi \rightarrow J^{s} \pi, \pi^{k}: J^{k} \pi \rightarrow E, \bar{\pi}^{k}: J^{k} \pi \rightarrow M$; for $k>s \geq 0$. As above, natural coordinates in $J^{2 k-1} \pi$ are $\left(t, q_{i}^{\alpha}\right) ; 0 \leq i \leq 2 k-1,1 \leq \alpha \leq n$. The (only) total time derivative is

$$
D_{t}=\frac{\partial}{\partial t}+\sum_{i=0}^{k} q_{i+1}^{\alpha} \frac{\partial}{\partial q_{i}^{\alpha}}
$$


which verifies the properties stated in Section 2, The dynamics is given by a Lagrangian form $\mathcal{L} \in \Omega^{1}\left(J^{k} \pi\right)$, which is a $\bar{\pi}^{k}$-semibasic 1-form and it has associated the Lagrangian function $L \in \mathrm{C}^{\infty}\left(J^{k} \pi\right)$, such that $\mathcal{L}=L\left(\bar{\pi}^{k}\right)^{*} \mathrm{~d} t$, where $\mathrm{d} t$ is the canonical volume form in $\mathbb{R}[6]$. The Poincaré-Cartan 1-form $\Theta_{\mathcal{L}} \in \Omega^{1}\left(J^{2 k-1} \pi\right)$ is given locally by:

$$
\Theta_{\mathcal{L}}=\sum_{r=1}^{k} L_{\alpha}^{r} \mathrm{~d} q_{r-1}^{\alpha}+\left(L-\sum_{r=1}^{k} L_{\alpha}^{r} q_{r}^{\alpha}\right) \mathrm{d} t,
$$

where the functions $L_{\alpha}^{r} \in C^{\infty}\left(J^{2 k-1} \pi\right)$ are

$$
L_{\alpha}^{r}=\sum_{i=0}^{k-r}(-1)^{i} D_{t}^{i}\left(\frac{\partial L}{\partial q_{r+i}^{\alpha}}\right),
$$

and they can be obtained inductively by setting $L_{\alpha}^{r}=0$, for $r>k$, and

$$
L_{\alpha}^{r}=\frac{\partial L}{\partial q_{r}^{\alpha}}-D_{t} L_{\alpha}^{r+1}
$$

The properties stated in Lemma 1 and Propositions 1 and 2 read:

Lemma 2. For $s \geq k-1$, the following conditions are equivalent:

1. $\Theta_{\mathcal{L}}$ projects onto $J^{s} \pi$.

2. $\mathrm{d} \Theta_{\mathcal{L}}$ is $\pi_{s}^{2 k-1}$-semibasic.

3. $\mathrm{L}(X) L_{\alpha}^{r}=0$; for every $X \in \mathfrak{X}^{V}\left(\pi_{s}^{2 k-1}\right)$, and for $r=1, \ldots, k, \alpha=1, \ldots, n$.

(Proof): $\quad(1 \Leftrightarrow 2)$ is a consequence of Cartan's formula. For the equivalence between 2 and 3 we consider two cases:

- If $s \geq k$ : The relevant terms of $\mathrm{d}_{\mathcal{L}}$ are of the form:

$$
\frac{\partial L_{\alpha}^{i}}{\partial q_{r}^{\beta}} \mathrm{d} q_{r}^{\beta} \wedge \mathrm{d} q_{i-1}^{\alpha} \quad, \quad \frac{\partial}{\partial q_{r}^{\beta}}\left(L-\sum_{i=1}^{k} L_{\alpha}^{i} q_{i}^{\alpha}\right) \mathrm{d} q_{r}^{\beta} \wedge \mathrm{d} t \quad ; \quad s<r \leq 2 k-1 .
$$

Then, $\mathrm{d} \Theta_{\mathcal{L}}$ is $\pi_{s}^{2 k-1}$-semibasic if, and only if, $\frac{\partial L_{\alpha}^{i}}{\partial q_{r}^{\beta}}=0$, and this is equivalent to $\mathrm{L}(X) L_{\alpha}^{r}=0$, for every $X \in \mathfrak{X}^{V}\left(\pi_{s}^{2 k-1}\right)$, since $\left\{\frac{\partial}{\partial q_{r}^{\beta}}\right\}$ generates $\mathfrak{X}^{V}\left(\pi_{s}^{2 k-1}\right)$.

- If $s=k-1$ : In this case $\mathrm{d} \Theta_{\mathcal{L}}$ is $\pi_{s}^{2 k-1}$-semibasic if, and only if,

$$
\frac{\partial L_{\alpha}^{i}}{\partial q_{r}^{\beta}}=0 \quad, \quad \frac{\partial L}{\partial q_{k}^{\beta}}-L_{\beta}^{k}=0 \quad ;
$$


but this last condition is fulfilled by the definition of $L_{\beta}^{k}$, and the same reasoning above allows us to prove the statement.

If $\Theta_{\mathcal{L}}$ projects onto $J^{s} \pi$, with $s<k-1$, then $L$ does not depend on $q_{j}^{\alpha}$, for $j>s+1$, then there exists a function $L^{\prime} \in C^{\infty}\left(J^{s+1} \pi\right)$ such that $L=\left(\pi_{s+1}^{k}\right)^{*} L^{\prime}$ and the theory is not strictly of order $k$. Furthermore, in the case $s \geq k-1$, a Lagrangian such that $\Theta_{\mathcal{L}}$ projects onto $J^{s} \pi$ depends on all the variables and thus we have a theory of order $k$, although the associated Euler-Lagrange equations are of lower order as a system of differential equations. In fact:

Proposition 3. If $\Theta_{\mathcal{L}}$ projects onto $J^{s} \pi$, then the order of the Euler-Lagrange equations is at most $s+1$.

(Proof): Note that $L_{\alpha}^{0} \in C^{\infty}\left(J^{2 k} \pi\right)$. For a curve $\phi \in \Gamma(\pi)$ which is a solution to the Euler-Lagrange equations we have that $\left.L_{\alpha}^{0}\right|_{j^{2 k-1} \phi}=0$. Then, for $X \in \mathfrak{X}^{V}\left(\pi_{s+1}^{k}\right)$,

$$
\mathrm{L}(X) L_{\alpha}^{0}=\mathrm{L}(X) \frac{\partial L}{\partial q_{0}^{\alpha}}-\mathrm{L}(X)\left(D_{t} L_{\alpha}^{1}\right)=\mathrm{L}(X) \frac{\partial L}{\partial q_{0}^{\alpha}}-D_{t}\left(\mathrm{~L}(X) L_{\alpha}^{1}\right)-\mathrm{L}\left(\left[X, D_{t}\right]\right) L_{\alpha}^{1} .
$$

Since $\left[D_{t}, X\right] \in \mathfrak{X}^{V}\left(\pi_{s}^{k}\right)$ and $L_{\alpha}^{1}$ and $L$ are $\pi_{s}^{k}$-basic, then $\mathrm{L}(X)\left(L_{\alpha}^{0}\right)=0$. Therefore, after evaluating on the section, the resulting equations only contain derivations up to order $s+1$.

Equating the local expressions of $\Theta_{\mathcal{L}}$ and $\Theta_{\mathcal{L}^{\prime}}$ the following result holds immediately:

Proposition 4. If there exist $\mathcal{L}^{\prime} \in \Omega^{1}\left(J^{k^{\prime}} \pi\right)$ such that $\Theta_{\mathcal{L}}=\left(\pi_{s}^{2 k-1}\right)^{*} \Theta_{\mathcal{L}^{\prime}}$, then $\mathcal{L}=\left(\pi_{s}^{2 k-1}\right)^{*} \mathcal{L}^{\prime}$

In particular $L$ is not strictly of order $k$.

Finally, a similar result to theorem 1 is the following:

Theorem 2. If $\Theta_{\mathcal{L}}$ projects onto $J^{s} \pi$, then solutions to the corresponding EulerLagrange equations exist only in points of a submanifold $\mathcal{S} \hookrightarrow J^{2 k-1} \pi$, where $\mathcal{S}$ is locally defined by the constraint functions given by

$$
D_{t}^{j} L_{\alpha}^{0}=0 \quad ; \quad(j=0, \ldots, 2 k-s-2) .
$$

(Proof): To find a solution to the Euler-Lagrange equations is equivalent to find a holonomic vector field $X \in \mathfrak{X}\left(J^{2 k-1} \pi\right)$ such that

$$
i(X) \mathrm{d} \Theta_{\mathcal{L}}=0
$$

The holonomic vector fields have the local expression:

$$
X=D_{t}+\left(F^{\alpha}-q_{2 k}^{\alpha}\right) \frac{\partial}{\partial q_{2 k-1}^{\alpha}},
$$


and then equation (12) reduces to

$$
L_{\alpha}^{0}-\left(F^{\beta}-q_{2 k}^{\beta}\right) \frac{\partial L_{\alpha}^{1}}{\partial q_{2 k-1}^{\beta}}=0 .
$$

If $\Theta_{\mathcal{L}}$ projects onto $J^{s} \pi$ for $s<2 k-1$, the second term vanishes and $L_{\alpha}^{0}=0$. Notice that we cannot compute any function $F^{\alpha}$. Actually $L_{\alpha}^{0} \in C^{\infty}\left(J^{2 k-1} \pi\right)$, thus $L_{\alpha}^{0}=0$ is just a restriction for the points of the manifold $J^{2 k-1} \pi$. Next, following the constraint algorithm [5], we impose the tangency condition and we get

$$
0=\mathrm{L}(X) L_{\alpha}^{0}=D_{t} L_{\alpha}^{0}+\left(F^{\alpha}-q_{2 k}^{\alpha}\right) \frac{\partial L_{\alpha}^{0}}{\partial q_{2 k-1}^{\alpha}} .
$$

If $\Theta_{\mathcal{L}}$ projects onto $J^{s} \pi$, then the second term vanishes (Proposition 3) and we find another constraint, $D_{t} L_{\alpha}^{0}=0$. The algorithm continues until we reach the condition $D_{t}^{2 k-s-2} L_{\alpha}^{0}=0$.

As above, depending on the Lagrangian, we may need to continue the constraint algorithm, obtaining that

$$
0=D_{t}\left(D_{t}^{2 k-s-2} L_{\alpha}^{0}\right)+\left(F^{\alpha}-q_{2 k}^{\alpha}\right) \frac{\partial}{\partial q_{2 k-1}^{\alpha}}\left(D_{t}^{2 k-s-2}\left(L_{\alpha}^{0}\right)\right) .
$$

This process continues until the new conditions hold identically.

\section{Acknowledgments}

We acknowledge the financial support of the Ministerio de Ciencia e Innovación (Spain), project MTM2014-54855-P, and of Generalitat de Catalunya, project 2014SGR-634. We want to thank to the referees for their valuable comments and suggestions that have allowed us to improve the final version of this work.

\section{References}

[1] V. Aldaya, J.A. de Azcárraga, "Higher order Hamiltonian formalism in Field Theory", J. Phys. A 13(8), 2545-255 (1980).

[2] S.M. Carroll, Spacetime and Geometry: An Introduction to General Relativity. Addison-Wesley, S. Francisco, 2004.

[3] M. Castrillón, J. Muñoz-Masqué, M.E. Rosado, "First-order equivalent to EinsteinHilbert Lagrangian", J. Math. Phys. 55(8), 082501 (2014).

[4] M. de León, J. Marín-Solano, J.C. Marrero, M.C. Muñoz-Lecanda, N. Román-Roy, "Premultisymplectic constraint algorithm for field theories", Int. J. Geom. Methods Mod. Phys. 2(5), 839-871 (2005). 
[5] M. de León, J. Marín-Solano, J.C. Marrero, "The constraint algorithm in the jet formalism", Diff. Geom. and Appls. 6(3), 275-300 (1996).

[6] M. de León, P.R. Rodrigues, Generalized classical mechanics and field theory, NorthHolland Math. Studies 112, Elsevier Science Publishers B.V., Amsterdam, 1985.

[7] A. Echeverría-Enríquez, M.C. Muñoz-Lecanda, N. Román-Roy, "Multivector fields and connections: Setting Lagrangian equations in field theories", J. Math. Phys. 39(9), 4578-4603 (1998).

[8] M. Francaviglia, D. Krupka, "The Hamiltonian formalism in higher order variational problems", Ann. Inst. H. Poincaré Sect. A (N.S.) 37(3), 295-315 (1982).

[9] P.L. García, J.M. Masque, "Le probleme de la regularité dans le calcul des variations du second ordre", C. R. Acad. Sci. Math. 301 639-642 E (1985).

[10] I. Kolár, "A geometrical version of the higher order Hamilton formalism in fibered manifolds", J. Geom. and Phys. 1(2), 127-137 (1984).

[11] S. Kouranbaeva, S. Shkoller, "A variational approach to second-order multisymplectic field theory", J. Geom. Phys. 35(4), 333-366 (2000).

[12] D. Krupka, "On the higher order Hamilton theory in fibered spaces", Procs. Conference on Differential Geometry and its Applications, Part 2, 167-183, Univ. J. E. Purkyne, Brno, 1984.

[13] D. Krupka, Introduction to Global Variational Geometry, Atlantis Studies in Variational Geometry, Atlantis Press 2015,

[14] D. Krupka, O. Stepankova, " On the Hamilton form in second order calculus of variations", Procs. Int. Meeting on Geometry and Physics, 85-101. Florence 1982, Pitagora, Bologna, 1983.

[15] P.D. Prieto Martínez, N. Román-Roy, "A new multisymplectic unified formalism for second-order classical field theories", J. Geom. Mech. 7(2), 203-253 (2015).

[16] M.E. Rosado, J. Muñoz-Masqué, "Integrability of second-order Lagrangians admitting a first-order Hamiltonian formalism", Diff. Geom. and Apps. 35 (Sup. September 2014), 164-177 (2014).

[17] M.E. Rosado, J. Muñoz-Masqué, "Second-order Lagrangians admitting a first-order Hamiltonian formalism", ArXiv: 1509.01037 [math.ph] (2015).

[18] D.J. Saunders, The geometry of jet bundles, London Mathematical Society, Lecture Notes Series 142, Cambridge Univ. Press, Cambridge, New York 1989.

[19] D.J. Saunders, M. Crampin, "On the Legendre map in higher-order field theories", $J$. Phys. A: Math. Gen. 23(14), 3169-3182 (1990).

[20] D. Vey, "Multisymplectic formulation of vielbein gravity. De Donder-Weyl formulation, Hamiltonian ( $n-1)$-forms", arXiv: 1404.3546 [math-ph] (2015). 